\title{
Accelerated SuFEx Click Chemistry for Modular Synthesis
}

\author{
Christopher J. Smedley ${ }^{\dagger[b]}$, Timothy L. Gialelis ${ }^{\dagger[b]}$ and John E. Moses ${ }^{*[a][b]}$
}

\begin{abstract}
Click chemistry is a method for the rapid synthesis of functional molecules with desirable properties. We report the development of accelerated SuFEx, a powerful click reaction for the efficient coupling of aryl and alkyl alcohols directly with SuFExable hubs catalyzed by 2-tert-butyl-1,1,3,3-tetramethylguanidine (BTMG, Barton's base). The new method circumvents the need to synthesize silyl ether substrates while allowing the use of sub-stoichiometric catalyst loadings. This is made possible through a synergistic effect between BTMG and hexamethyldisilazane (HMDS) additive. The powerful combination drives the in situ formation of reactive TMSether intermediates while exploiting the silicon-fluoride bond formation's thermodynamic driving force. Comparatively, the required BTMG base's catalyst loading is generally low $(1.0-20 \mathrm{~mol} \%)$ compared to the dominant SuFEx catalyst, DBU (10-30 mol\%). In line with click chemistry principles, the scalable reaction only requires simple evaporation of the volatile side products $\left(\mathrm{NH}_{3}, \mathrm{Me}_{3} \mathrm{Si}-\mathrm{F}\right.$, TMS$\mathrm{OH}, \mathrm{BTMG}$ ) under reduced pressure instead of extensive purification. The new SuFEx protocol is tolerant of a wide selection of functional groups and meets all the demands of a click reaction, thereby dramatically shortening reaction times and delivering products in excellent yield.
\end{abstract}

The Sulfur(VI) Fluoride Exchange (SuFEx) ${ }^{[1,2]}$ reaction, first developed by Sharpless and co-workers in 2014, represents a new class of ideal click chemistry transformations ${ }^{[3-5]}$ that are wide in scope and application ${ }^{[5]}$

Classic SuFEx reactions involve substituting a stable SVI-F bond with aryl silyl ethers to give the corresponding $\mathrm{S}^{\mathrm{VI}}-\mathrm{O}$ union (Figure 1). However, S-F exchange also occurs with amines ${ }^{[6-8]}$, organometallic reagents ${ }^{[9]}$, and other carbon nucleophiles ${ }^{[10]}$ to yield stable $\mathrm{S}^{\mathrm{VI}}-\mathrm{N}$ and $\mathrm{S}^{\mathrm{V}}-\mathrm{C}$ bonds, respectively. The operational simplicity and robust nature of SuFEx, coupled with the availability of commercial SuFEx substrates (alcohols, amines, etc.), renders this modern click-reaction perfect for modular synthesis ${ }^{[11]}$ and for accessing diverse click libraries ${ }^{[12]}$.

Another essential feature unique to SuFEx is the growing number of versatile SuFExable hubs - typified by $\mathrm{SO}_{2} \mathrm{~F}_{2}, \mathrm{SOF}_{4}$, ESF, BESF, and SASFs ${ }^{[1,13-18]}$ - serving as powerful connectors for creating diverse functional molecules and broadening the ever-expanding applications of click chemistry (Figure 1B) ${ }^{[18]}$.

Key to SuFEx reactivity is the requirement for fluoride to transit from a strong covalent S-F bond to a leaving group; a process that can be assisted by interactions with $\mathrm{H}^{+}$or $\mathrm{R}_{3} \mathrm{Si}^{+}$, and mediated by

[a] Prof. Dr. J. E. Moses, Cancer Center, Cold Spring Harbor Laboratory, 1 Bungtown Road, Cold Spring Harbor, NY 11724, USA E-mail: moses@cshl.edu

[b] Dr. C. J. Smedley, T. L. Gialelis, La Trobe Institute for Molecular Science, Melbourne, VIC 3086, Australia

$\dagger \quad$ These authors contributed equally.

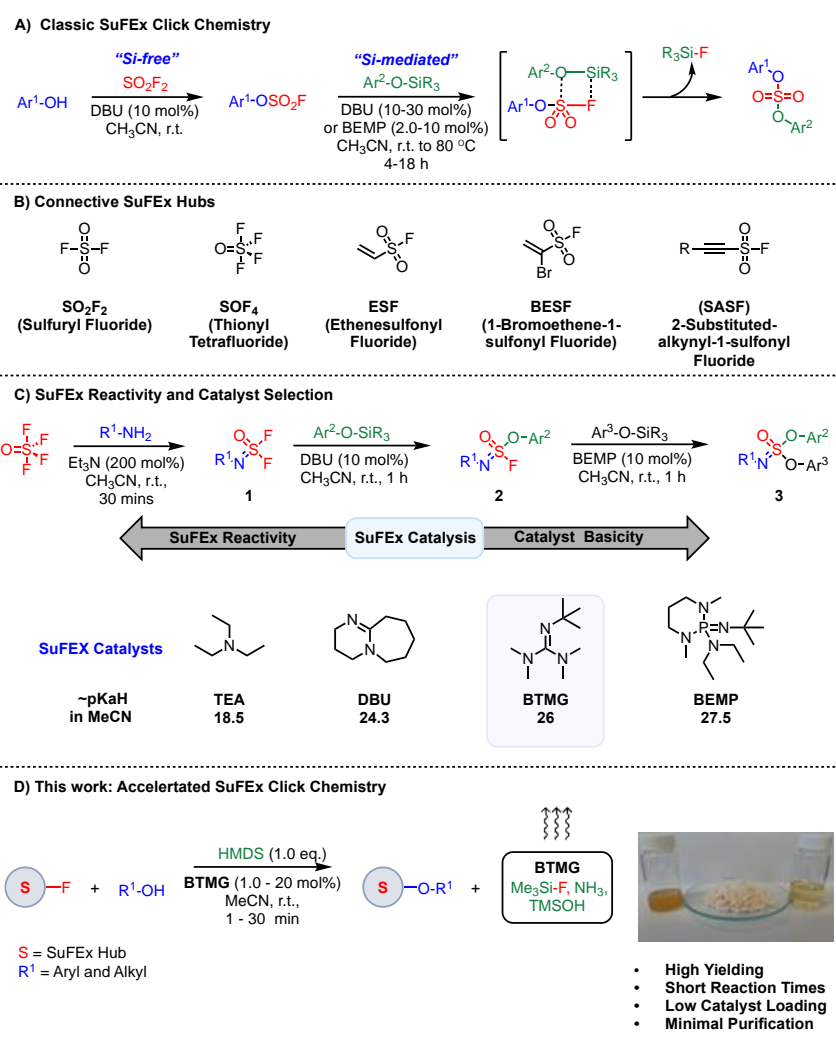

Figure 1. A) Example of classic Si-free and Si-mediated SuFEx click reactions. B) Examples of connective SuFEx hubs for modular click chemistry. C) The relationship between SuFExability of $S^{V I}-F$ electrophiles and catalyst activity. D) The development of Accelerated SuFEx Click Chemistry: BTMG catalyzed coupling of aryl and alkyl alcohols with SuFExable hubs mediated by HMDS.

catalysts including basic tertiary amines (e.g., $\mathrm{Et}_{3} \mathrm{~N}$ ), amidines (e.g., DBU), phosphazenes (BEMP), and bifluoride ion salts ${ }^{[1,19-}$ 23].

SuFEx catalyst selection is influenced by the $\mathrm{S}^{\mathrm{VI}}-\mathrm{F}$ sulfur core's electrophilicity, which is a measure of the functional group's relative SuFExability. The series of SuFEx reactions from $\mathrm{SOF}_{4}$, illustrated in Figure 1C, exemplifies this trend. The reactive $\mathrm{SOF}_{4}$ gas first undergoes rapid SuFEx with primary amines under $\mathrm{Et}_{3} \mathrm{~N}$ catalysis to give the iminosulfur oxydifluorides 1 ; subsequent and sequential SuFEx of the remaining $S^{V^{V}}-F$ bonds (i.e., $\mathbf{1} \rightarrow \mathbf{2} \rightarrow \mathbf{3}$ ) require increasingly stronger DBU and BEMP catalysis, respectively (Figure $1 \mathrm{C})^{[13]}$. While steric factors play an important role in catalyst function, the relative basicity is key to catalyst activity; the stronger the base, the more active the catalyst (e.g., $\mathrm{Et}_{3} \mathrm{~N}<\mathrm{DBU}<\mathrm{BEMP}$, Figure 1C). Hence, through judicious choice of reaction conditions, the relationship between SuFExability and catalyst activity can endow remarkable chemoselectivity for orthogonal $\mathrm{S}^{\mathrm{VI}}$-F functionality (Figure 1C).

The fidelity and versatility of SuFEx secure its place as one of the most perfect click reactions to date; nevertheless, there is still a margin for operational improvement. For example, the required SuFEx catalyst loadings are often in the range of between $5.0-20 \mathrm{~mol} \%$. Loadings can be even higher ${ }^{[23]}$ when the 
Table 1. Table of optimization for Accelerated SuFEx Click Chemistry.

\begin{tabular}{|c|c|c|c|c|}
\hline 4 & 5 & $\begin{array}{c}\text { Si-Source }(X \text { eq. }) \\
\text { Catalyst }(Y \text { mol\%) } \\
\mathrm{T}(\mathrm{min}) \\
\mathrm{MeCN} \text {, r.t. }\end{array}$ & \multicolumn{2}{|c|}{6} \\
\hline Entry & $\begin{array}{l}\text { Si-X Source } \\
\text { (eq.) }\end{array}$ & $\begin{array}{l}\text { Catalyst } \\
(\mathrm{mol} \%)\end{array}$ & $\mathrm{T}(\min )$ & $\begin{array}{c}\text { Yield } \\
(\%)\end{array}$ \\
\hline 1 & - & DBU (20) & 30 & $20^{[b]}$ \\
\hline 2 & TMSOH (1.0) & DBU (20) & 30 & 21 \\
\hline 3 & $(\mathrm{TMS})_{2} \mathrm{O}(1.0)$ & DBU (20) & 30 & 97 \\
\hline 4 & HMDS (1.0) & DBU (20) & 1 & $>98$ \\
\hline 5 & $\begin{array}{l}\text { Hexamethyldisilane } \\
\text { (1.0) }\end{array}$ & DBU (20) & 30 & $61^{[\mathrm{b}]}$ \\
\hline 6 & HMDS (1.0) & BTMG (20) & 1 & $>99$ \\
\hline 7 & HMDS (1.0) & BTMG (10) & $<5$ & $>99$ \\
\hline 8 & HMDS (1.0) & BTMG (5) & $<5$ & $>99$ \\
\hline 9 & HMDS (1.0) & BTMG (1.0) & $<5$ & $>99$ \\
\hline 10 & HMDS (1.0) & BTMG (0.5) & 30 & 96 \\
\hline 11 & HMDS (1.0) & BTMG (0.1) & 30 & 0 \\
\hline 12 & HMDS (0.5) & BTMG (1.0) & 30 & $66^{[b]}$ \\
\hline
\end{tabular}

[a] Reactions were performed on $0.1 \mathrm{mmol}$ scale and stirred for $30 \mathrm{~min}$ or until complete conversion by TLC; [b] Conversion determined by ${ }^{1} \mathrm{H}$ NMR. reaction conditions result in catalyst degradation, such as the hydrolysis of DBU[24].

Another factor affecting the rate of SuFEx reactions is the steric bulk around the silicon center. Generally, smaller groups like trimethylsilyl ethers react rapidly, whereas bulkier tertbutyldimethylsilyl groups can require several hours for the reaction to reach completion ${ }^{[1]}$. Nevertheless, the bulkier silyl ether modules are more frequently used in SuFEx applications due to their superior bench-stability.

Several prototypical examples of silicon-free SuFEx reactions with aryl alcohols ${ }^{[1,25,26]}$ that negate the need to prepare the silyl ethers are known. Eliminating synthetic steps is practical and has environmental and economic benefits, particularly when synthesizing large libraries of compounds ${ }^{[12]}$. However, these Sifree reactions do not profit from the formation of the thermodynamically favorable Si-F bond $\left(B D E=135 \mathrm{kcal} \mathrm{mol}^{-1}\right)$, and the benefit of using the alcohol substrate directly is often outweighed by the need for higher catalyst loadings, leaving much scope for improvement.

Seeking to overcome the issues of catalyst degradation and exploit native alcohol substrates directly in SuFEx reactions, we report the development of Accelerated SuFEx Click Chemistry. For the first time, near-perfect SuFEx catalysis is achieved by the sterically hindered 2-tert-butyl-1,1,3,3-tetramethylguanidine base (Barton's base, BTMG) ${ }^{[27,28]}$, that, in concert with the silicon additive hexamethyldisilazane (HMDS), deliver SuFEx products in high yield within minutes. The broad-spectrum reaction outperforms existing SuFEx protocols at lower catalyst loadings while simultaneously eliminating the need for preparing silyl ether substrates.

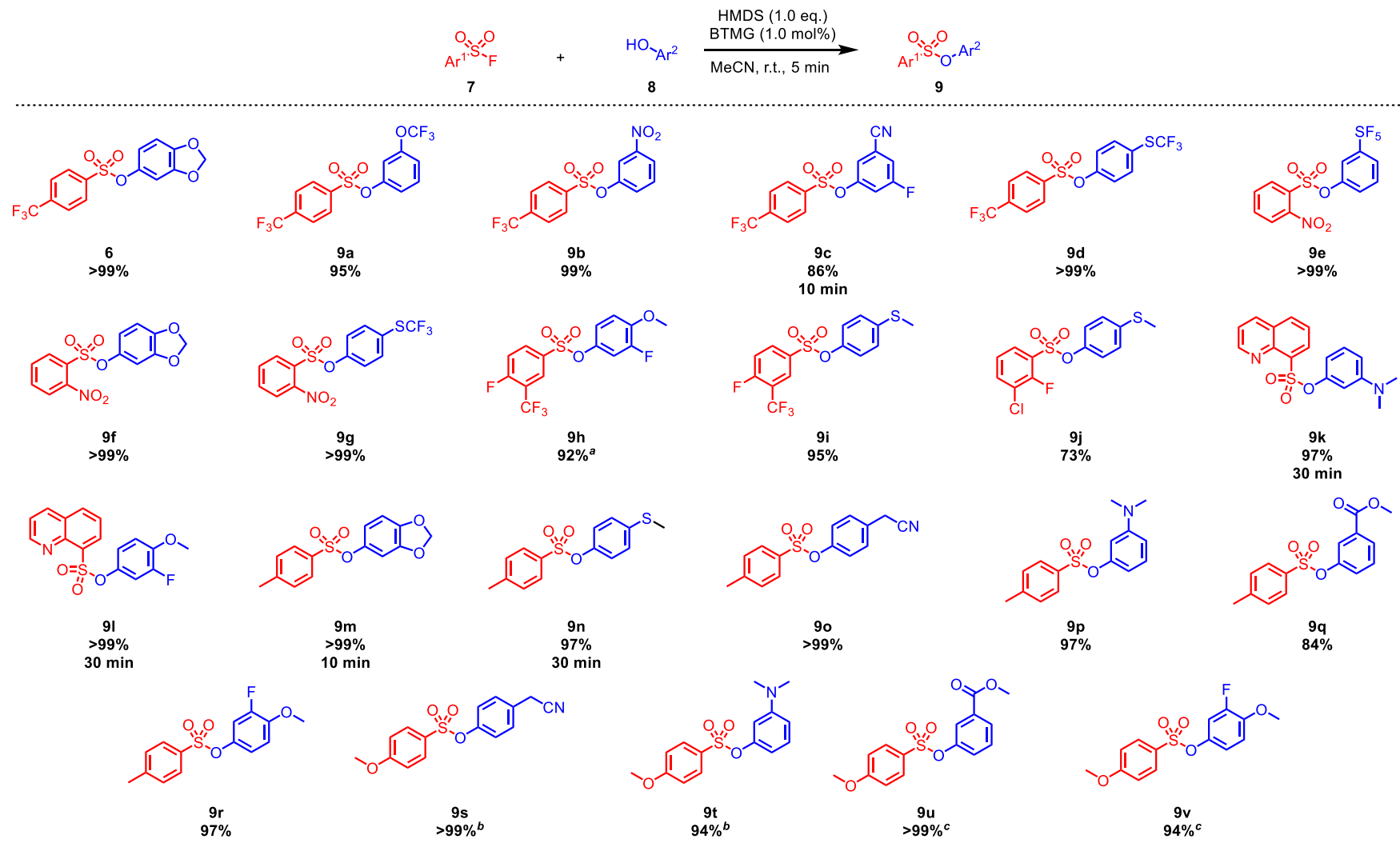

Scheme 1. BTMG catalyzed SuFEx reactions between aryl sulfonyl fluorides $(7,0.1 \mathrm{mmol})$ and aryl alcohols $(8,0.1 \mathrm{mmol})$ with $\mathrm{HMDS}(0.1 \mathrm{mmol})$, BTMG (1.0 $\mu \mathrm{mol})$ stirring for $5 \mathrm{~min}$. [a] $5.0 \mathrm{~mol} \%$ BTMG, $30 \mathrm{~min}$; [b] $10 \mathrm{~mol} \%$ BTMG, $30 \mathrm{~min}$; [c] $10 \mathrm{~mol} \%$ BTMG, $60 \mathrm{~min}$. 


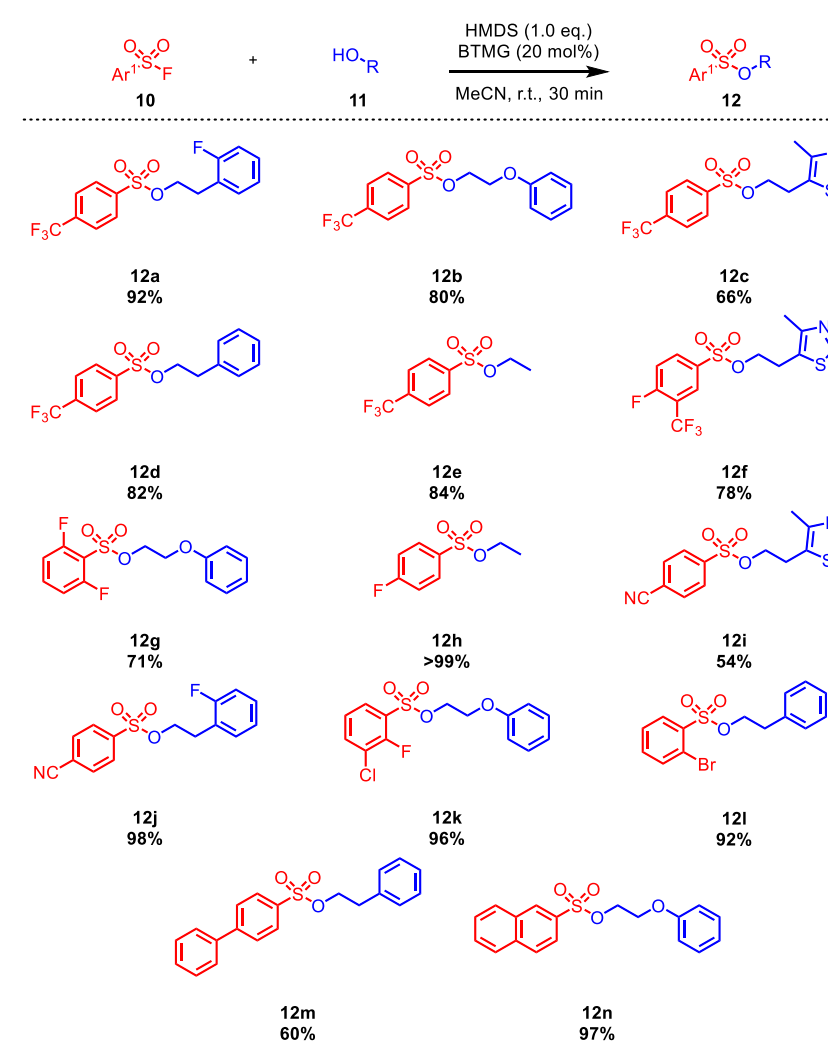

Scheme 2. BTMG catalyzed SuFEx between aromatic sulfonyl fluorides (10 $0.1 \mathrm{mmol})$ and alkyl alcohols $(\mathbf{1 1}, 0.1 \mathrm{mmol})$ in MeCN with HMDS $(0.1 \mathrm{mmol})$ BTMG $(20 \mu \mathrm{mol})$ stirring for $30 \mathrm{~min}$.

Our studies began with a search for a suitable silicon additive that would work in synergy with a catalyst to activate the SuFEx process while also sequestering the fluoride ion product and minimizing the formation of HF. The ideal additive would serve two functions: 1) extend the catalyst's life and allow for lower loadings, and 2) enable alcohol substrates to be used directly, eliminating the need to synthesize silyl ether substrates.

A selection of silicon additives, including $\mathrm{TMSOH}^{[29]}$, hexamethyldisiloxane, hexamethyldisilane, and HMDS (Table 1, entries 2-7), were employed in the SuFEx reaction between 4(trifluoromethyl)benzenesulfonyl fluoride (4) and sesamol (5) using otherwise standard SuFEx conditions [20 mol\% DBU in reagent grade $\mathrm{MeCN}^{[30]}$. The SuFEx reactions were allowed to proceed for a maximum of 30 minutes, with their progress monitored by TLC. SuFEx coupling occurred in each case giving rise to the sulfonate product 6 with varied yields $(20-99 \%)$ at markedly different rates (Table 1, entries 1-5). The control reaction without additive (entry 1 ) and a reaction with $\mathrm{TMSOH}$ as an additive (entry 2 ) performed least well, giving $20 \%$ and $21 \%$ of the sulfonate 6 , respectively. The reaction with HMDS was complete within less than one minute and giving quantitative conversion to 6 (Table 1, entry 4), while hexamethyldisilane performed less well (Table 1, entry 5). Niu and co-workers recently reported the SuFEx O-sulfation between aryl fluorosulfates and silylated hydroxyl groups of a selection of monosaccharides, disaccharides, and steroids under identical conditions [HMDS 1.0 eq. and $20 \mathrm{~mol} \% \mathrm{DBU}]^{[31]}$. A one-pot procedure was demonstrated, whereby the $O$-silylation by HMDS was presumed to occur in situ, followed by the subsequent SuFEx coupling. We, therefore, elected to exploit HMDS in the further

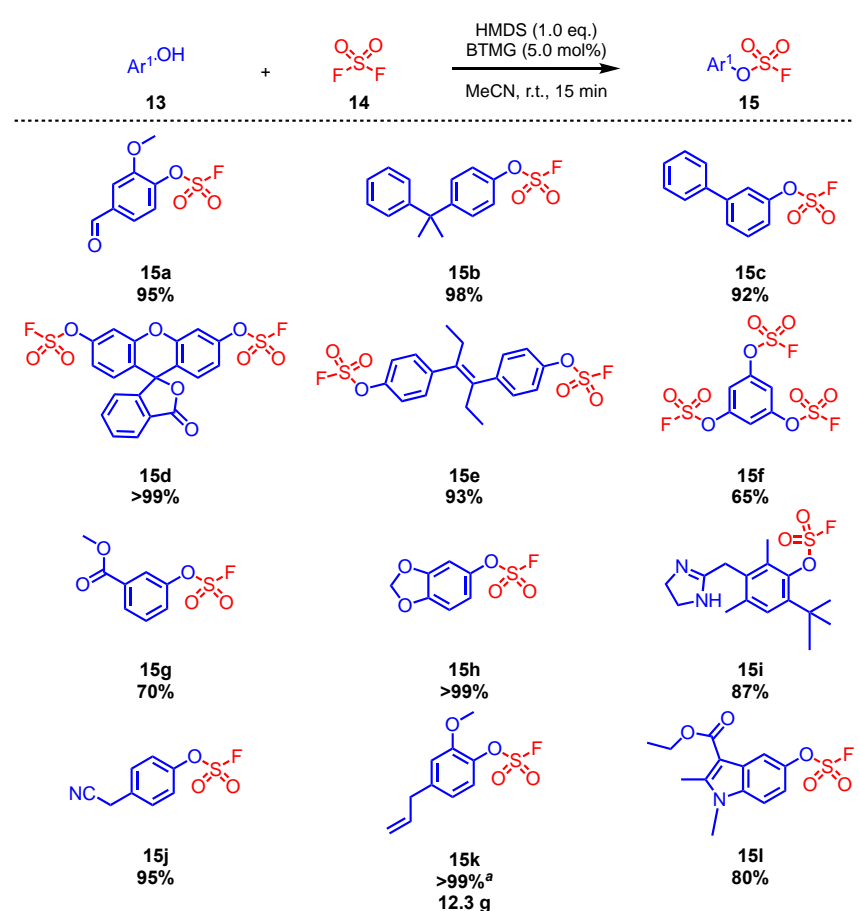

Scheme 3. BTMG catalyzed SuFEx between sulfuryl fluoride (14, balloon) and aryl alcohols (13, $0.1 \mathrm{mmol})$ in MeCN with $\operatorname{HMDS}(0.1 \mathrm{mmol}), \mathrm{BTMG}(5.0 \mu \mathrm{mol})$ stirring for $15 \mathrm{~min}$. [a] for large scale sulfuryl fluoride (14, balloon) and eugenol (13k, $50 \mathrm{mmol}$ ) with HMDS (50 mmol), BTMG (2.5 mmol) stirring for $5 \mathrm{~min}$.

development of accelerated SuFEx click chemistry.

Several catalysts have been deployed in SuFEx chemistry in recent years, including salts of bifluoride, phosphazene super bases (e.g., BEMP), along with more commonly used nitrogen bases like $\mathrm{DBU}^{[2]}$. However, to the best of our knowledge, the sterically hindered guanidine base derivatives originally developed by Barton in the 1980s have been overlooked in the context of SuFEx catalysis ${ }^{[32]}$. Recent reports of the utility of 2tert-butyl-1,1,3,3-tetramethylguanidine (BTMG, Barton's base) in combinatorial synthesis highlight its strength, volatility, and ease of use, rating it superior to traditional hindered organic bases such as $\mathrm{DBU}^{[33-35]}$.

BTMG is also known to generate phenolate anions effectively ${ }^{[27,28]}$ with a pKaH of the order of $\sim 26$ (in MeCN) ${ }^{[36]}$, that sits comfortably between $\mathrm{DBU}(\mathrm{pKaH}=24.3 \text { in } \mathrm{MeCN})^{[24]}$ and BEMP (pKaH $=27.6$ in $\mathrm{MeCN})^{[37]}$. We considered this a "sweet spot" that could provide a balance between reactivity and selectivity (Figure 1C).

Using various loadings of BTMG $(0.1,0.5,1.0,5.0$, and 10 mol\%) in acetonitrile, we explored the HMDS mediated SuFEx coupling between the model substrates 4 and $\mathbf{5}$. At $10 \mathrm{~mol} \%$, the BTMG catalysis resulted in total consumption of substrates within $5 \mathrm{~min}$; the same was observed with $5.0 \mathrm{~mol} \%$ (Table 1, entries 7 \& 8). Even with a loading of just $1.0 \mathrm{~mol} \%$ BTMG (Table 1, entry 9 ), the reaction reached completion within just 5 minutes. In contrast, the analogous reaction with DBU was notably sluggish, yielding $67 \%$ product 6 after $180 \mathrm{~min}$ (see SI, T1). While the lower loading of 0.5 mol\% BTMG gave the SuFEx product 6 (Table 1 , entry 10$)$, but the reaction was markedly slower. No reaction was observed with $0.1 \mathrm{~mol} \%$ BTMG after 30 minutes (Table 1, entry 11); hence $1.0 \mathrm{~mol} \%$ was deemed optimal. We find that the reaction with 0.5 equivalents of HMDS is also viable (Table 1 , entry 12), but the slower rate and lower product yield compelled us to advance using 1.0 equivalent for development. 


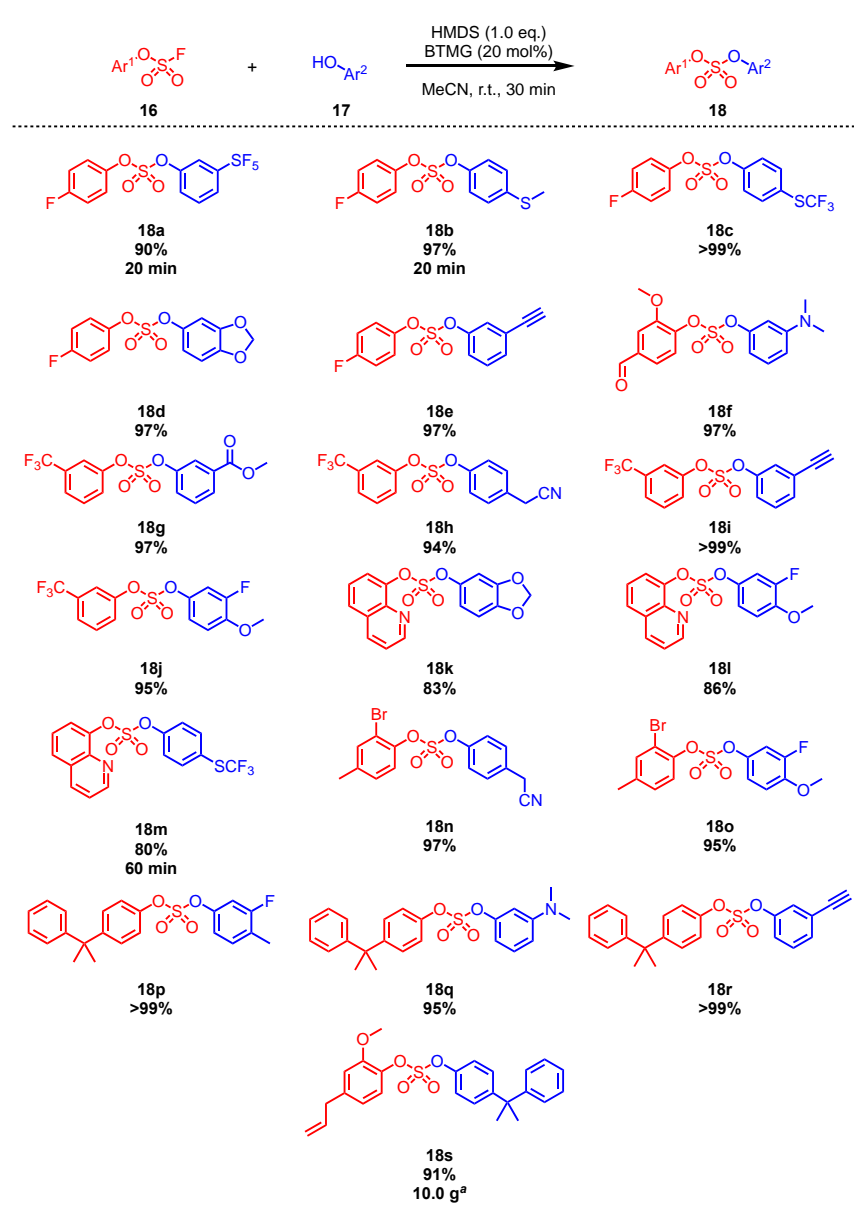

Scheme 4. BTMG catalyzed SuFEx between aryl fluorosulfates $(16,0.1 \mathrm{mmol})$ and aryl alcohols $(\mathbf{1 7}, 0.1 \mathrm{mmol})$ in MeCN with HMDS (0.1 mmol), BTMG (20 $\mu \mathrm{mol})$ stirring for $30 \mathrm{~min}$. [a] for large scale fluorosulfate $(\mathbf{1 6}, 25 \mathrm{mmol})$ and alcohol $(17,25 \mathrm{mmol})$ with HMDS (25 mmol), BTMG (5.0 mmol) stirring for 180 $\min$.

We next explored the substrate scope with a selection of aromatic sulfonyl fluorides and aryl alcohols. The exchange between electron-poor sulfonyl fluorides and both electron-poor and electron-rich aryl alcohols proceed smoothly under the new BTMG accelerated conditions; most proceeding to full completion within less than 5 minutes to give the sulfonate products $9 a-9 v$ in excellent isolated yield. In each case, the products were quickly recovered by removing the volatile by-products (e.g., BTMG, TMS-F, $\mathrm{NH}_{3} \& \mathrm{TMSOH}^{[38]}$ ) under reduced pressure or merely passing the reaction mixture through a short pad of silica. In some instances, with electron-rich sulfonyl fluoride substrates, increased catalyst loadings and reaction times were required (e.g. $9 \mathrm{~h}, 9 \mathrm{~s}-9 \mathrm{v})$. The method also works well with nitrogen heterocyclic sulfonyl fluorides (e.g., products $9 \mathbf{k}$ and $\mathbf{9 l}$, Scheme 1 ).

SuFEx reactions with alkyl alcohol nucleophiles are rare and more challenging than with aryl alcohols, not least due to competing $\mathrm{S}_{\mathrm{N}} 2$ pathways of the sulfonate products ${ }^{[39]}$. Nevertheless, we find that SuFEx reactions between aromatic sulfonyl fluorides and alkyl alcohols proceed smoothly at room temperature under the HMDS-BTMG protocol, albeit with an elevated catalyst loading of $20 \mathrm{~mol} \%$. The transformations are generally complete within 30 minutes, giving the sulfonate products $12 \mathbf{a}-12 \mathbf{n}$ in excellent yield and purity (Scheme 2 ).

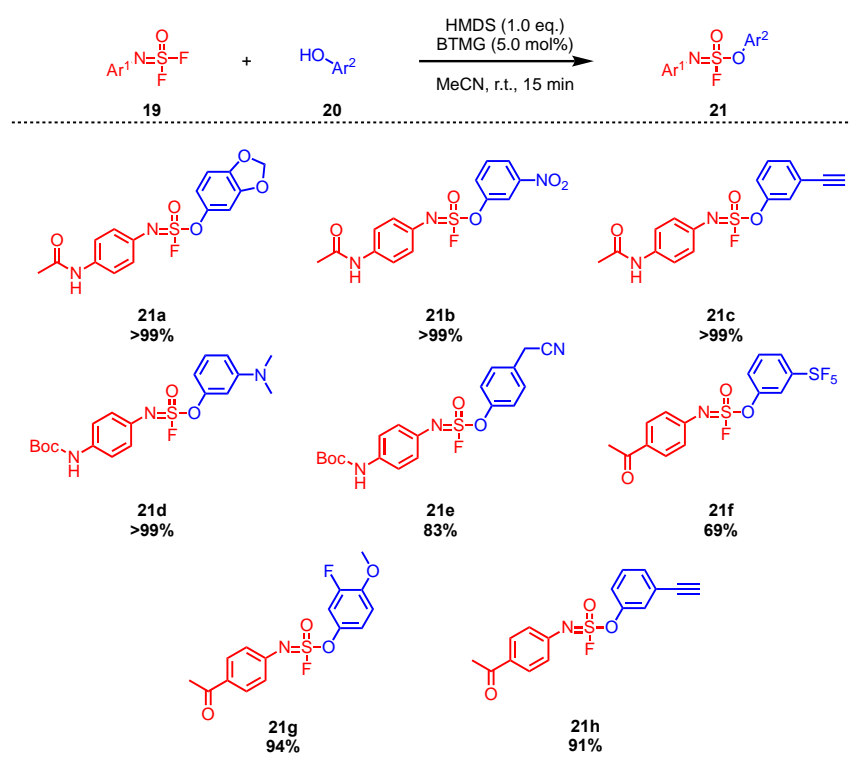

Scheme 5. BTMG catalyzed SuFEx between iminosulfur oxydifluorides $(\mathbf{1 9}, 0.1$ $\mathrm{mmol})$ and aryl alcohols $(20,0.1 \mathrm{mmol})$ in $\mathrm{MeCN}$ with HMDS $(0.1 \mathrm{mmol})$, BTMG $(5.0 \mu \mathrm{mol})$ stirring for $5 \mathrm{~min}$.

The sulfuryl fluoride $\left(\mathrm{SO}_{2} \mathrm{~F}_{2}\right)$ connective hub was next investigated as a substrate for the new protocol. Under classic SuFEx conditions, the reaction between $\mathrm{SO}_{2} \mathrm{~F}_{2}$ and aryl alcohols to the corresponding fluorosulfates requires at least 1.5 equivalents of the given base catalyst (e.g., $\left.\mathrm{Et}_{3} \mathrm{~N}\right)$, with reaction times between 2-6 hours. Under accelerated SuFEx conditions, with a slightly higher loading of BTMG $(5.0 \mathrm{~mol} \%)$, we find that reactions are complete within 15 minutes to deliver the fluorosulfates (15a-15I) in excellent yield. Particularly noteworthy are the syntheses of the fluorosulfate derivates of the drugs diethylstilbestrol (15e), oxymetazoline (15i), and mecarbinate (15I). The challenging benzene-1,3,5-triyl tris(sulfurofluoridate) (15f) is also accessible in good yield directly from the benzene1,2,3-triol. The previous approach to $\mathbf{1 5 f}$ first necessitated the synthesis of the corresponding TMS-ether substrate, along with prolonged reaction times $(4 \mathrm{~h})$ and high catalyst loadings (30 mol\% DBU $)^{[1]}$. The accelerated SuFEx protocol is also amenable to scale-up, exemplified by the $50 \mathrm{mmol}$ scale synthesis of the fluorosulfate (15k) from eugenol (Scheme 3).

Fluorosulfates are themselves valuable substrates for SuFEx, albeit significantly less reactive than their aryl sulfonyl fluoride counterparts. With a BTMG catalyst loading of $5.0 \mathrm{~mol} \%$, the coupling of aryl fluorosulfates and aryl alcohols proceed with good conversions at room temperature but require longer reaction times of up to $24 \mathrm{~h}$. However, increasing the catalyst loading to $20 \mathrm{~mol} \%$ results in total consumption of the fluorosulfate starting material within 30 minutes, giving the corresponding diaryl sulfates in excellent yield - even at scale (18s) (Scheme 4).

The multidimensional $\mathrm{SOF}_{4}$ derived iminosulfur oxydifluorides hubs (19) also work well. The SuFEx coupling of a range of aryl alcohols (20) proceeds to full completion within 15 minutes with a catalyst loading of just $5.0 \mathrm{~mol} \%$ at room temperature. This is a significant improvement over classic SuFEx with DBU, which requires loadings of between 10-20 mol\% and a reaction time of over $1 \mathrm{~h}$ with the aryl silyl ether equivalent substrates. 
The reaction was chemoselective, and we did not observe any competitive SuFEx coupling with the remaining S-F bond of the sulfurofluoridoimidate products $(\mathbf{2 1})^{[13]}$ (Scheme 5$)$.

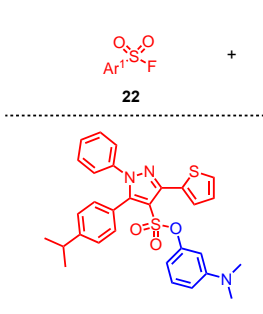

${ }_{76 \%}^{24 a}$

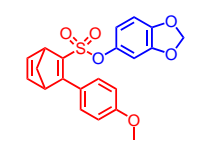

${ }_{88 \%}^{24 d}$

$\frac{24 \mathrm{~g}}{81 \%}$

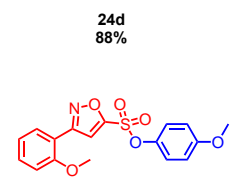

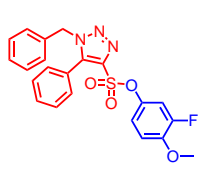

${ }_{73 \%}^{24 b}$

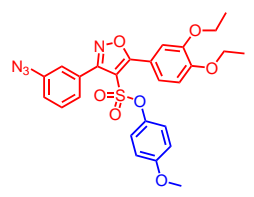

$83 \%$

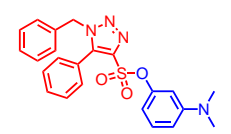

${ }_{80 \%}^{24 c}$

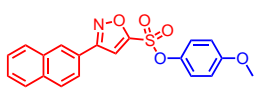

$24 \mathrm{f}$

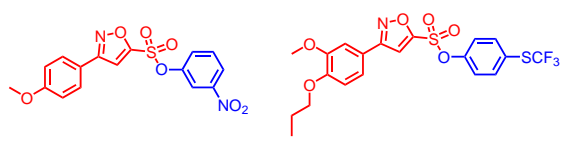

$\underset{>99 \%}{24 i}$
Scheme 6. BTMG catalyzed SuFEx reactions between SASF derived sulfonyl fluoride hubs (22a-22i, $0.1 \mathrm{mmol})$ and aryl alcohols $(\mathbf{2 3}, 0.1 \mathrm{mmol})$ with HMDS $(0.1 \mathrm{mmol})$, BTMG $(5.0 \mu \mathrm{mol})$ stirring for $5 \mathrm{~min}$.
Finally, a selection of diverse sulfonyl fluoride hubs derived from the Diversity Oriented Clicking (DOC) of BESF ${ }^{[15]}$ and SASF ${ }^{[18]}$; including pyrazole (22a), 1,2,3-triazoles (22b and 22c), diene (22d), and isoxazoles (22e-22l), were explored using the new method (Scheme 6). The aromatic heterocyclic sulfonyl fluoride substrates are challenging substrates for SuFEx, often requiring high $\mathrm{DBU}$ catalyst loadings and long reaction times with aryl silyl ether substrates. Under the new accelerated SuFEx conditions, we find that with a catalyst loading of just $5.0 \mathrm{~mol} \%$, the reactions between 22a-22i with a range of aryl alcohols proceed to completion within 5 minutes to give the corresponding aryl sulfonate derivatives (24a-24i) with good yields.

We next performed SuFEx between quinoline-8-sulfonyl fluoride (7k) and 3-(dimethylamino)phenol (8k) in deuterated acetonitrile to monitor the relatively slow reaction by ${ }^{1} \mathrm{H}$ NMR. Figure 2 illustrates the time-course of the reaction, which begins by the formation of the TMS-ether $(\mathbf{2 5})^{[31,40]}$ upon mixing $\mathbf{8 k}$ and $\mathrm{HMDS}^{[41]}$. As anticipated, the addition of the sulfonyl fluoride $\mathbf{7 k}$ gave no observable reaction until the BTMG catalyst (1.0 mol\%) was introduced. Monitoring the reaction by ${ }^{1} \mathrm{H}$ NMR at regular intervals clearly shows the smooth and clean conversion of $\mathbf{7 k}$ and $\mathbf{8 k}$ into the sulfonate $\mathbf{9 k}$.

While the mechanistic details of accelerated SuFEx are not yet fully understood, a simplistic rationale to account for the involvement of both HMDS and BTMG is presented in Scheme 7. Using the coupling of $\mathbf{7 k}$ and $\mathbf{8} \mathbf{k}$ as an example, the reaction begins with in situ formation of the TMS-ether 25 upon the reaction of the alcohol $\mathbf{8 k}$ and HMDS. BTMG may play a role as a catalyst in the formation of $\mathbf{2 5}$ via the reactive complex 26 .

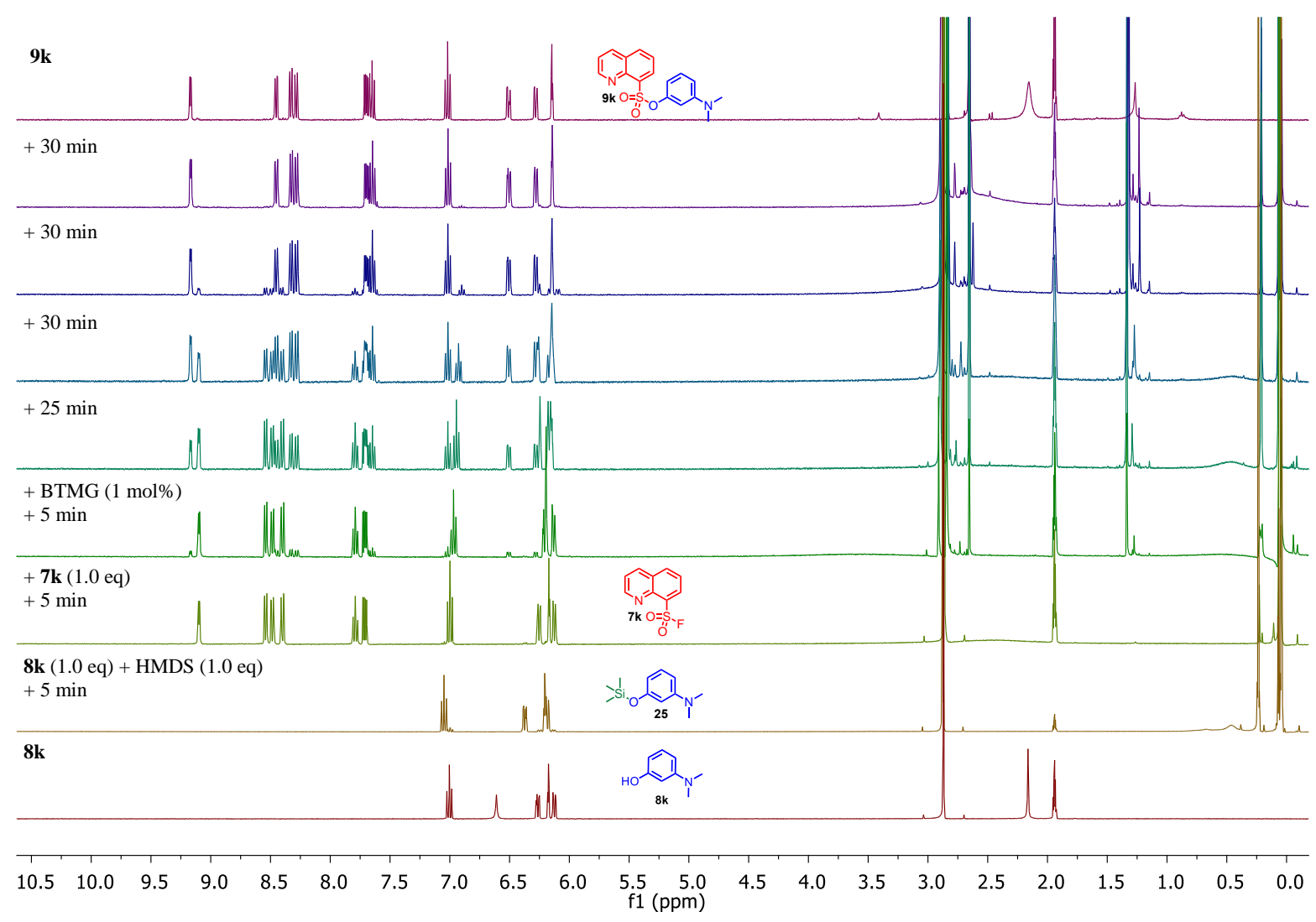

Figure 2. The HMDS-BTMG mediated accelerated SuFEx reaction between $\mathbf{7 k}$ with $\mathbf{8 k}$ monitored by ${ }^{1} \mathrm{H} N M R\left(C D_{3} C N\right)$. 


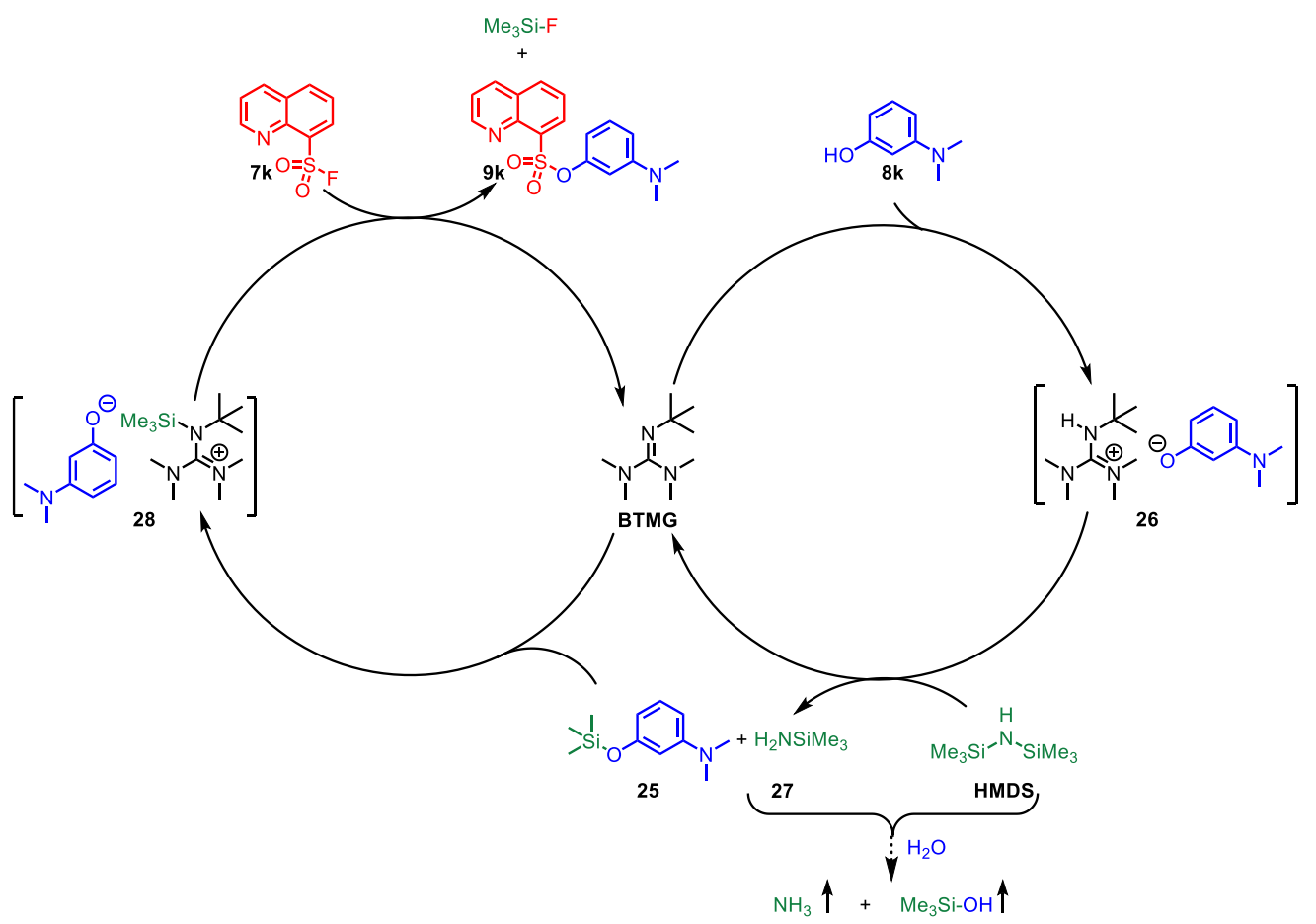

Scheme 7. A suggested working mechanism for the synergistic effect of the BTMG-HMDS accelerated SuFEx reaction.

Under classic SuFEx conditions, it is supposed that fluoride $\left(\mathrm{F}^{-}\right)$ and/or bifluoride ([FHF]') ion deprotect aryl silyl ether substrates to give reactive phenolates ${ }^{[25]}$; but this is unlikely in the presence of the fluoride sequestering silicon additive HMDS. We posit an alternative pathway involving the BTMG ${ }^{[42]}$ mediated de-silylation of 25 to the reactive guanidinium complex $28^{[43]}$ (plus the volatile by-products $\mathrm{NH}_{3}$ and $\mathrm{TMSOH}$ via hydration of 27 ), that itself undergoes immediate SuFEx with the sulfonyl fluoride $\mathbf{7 k}$ to yield the sulfonate product $\mathbf{9 k}$, TMS-F, and the regenerated BTMG catalyst.

To summarize, we introduce accelerated SuFEx as a nearperfect click reaction for the rapid coupling of alkyl and aryl alcohols with SuFExable hubs. For the first time, we demonstrate the hindered guanidine base BTMG (Barton's base) as a new class of SuFEx catalyst that, in concert with the silicon additive HMDS, functions as a uniquely powerful and robust accelerator of SuFEx across the board.

The accelerated SuFEx reactivity is achieved with relatively low catalyst loadings. It circumvents the need to prepare silylether substrates required in classic SuFEx click chemistry, thereby removing a bottle-neck in high-throughput synthesis. The reaction coupling partners are easily prepared or are widely available from natural resources and petroleum feedstocks with great abundance and structural diversity. We believe it would be of general interest for modular function-discovery in diverse fields.

\section{Acknowledgments}

We thank Cold Spring Harbor Laboratory for developmental funds from the $\mathrm{NCl}$ Cancer Center Support Grant 5P30CA045508 (JEM), the ARC (JEM) (Future Fellowship; FT170100156) for financial support. We thank Dr. A. S. Barrow for the fruitful discussions. We thank the Sharpless group (Scripps Research) for providing the substrates $19 a-h$.

\section{Conflict of interest}

The authors declare no conflicts of interest.

Keywords: Click Chemistry • Accelerated SuFEx • Catalysis • Barton's Base $\cdot$ Sulfonyl Fluorides

[1] J. Dong, L. Krasnova, M. G. Finn, K. B. Sharpless, Angew. Chem. Int Ed. 2014, 53, 9430-9448; Angew. Chem. 2014, 126, 9584-9603.

[2] A. S. Barrow, C. J. Smedley, Q. Zheng, S. Li, J. Dong, J. E. Moses, Chem. Soc. Rev. 2019, 48, 4731-4758.

[3] The term click chemistry was first coined by K. Barry Sharpless in 1998 and was later published as a concept in 2001 see ref: [4].

[4] H. C. Kolb, M. G. Finn, K. B. Sharpless, Angew. Chem. Int. Ed. 2001, 40, 2004-2021; Angew. Chem. 2001, 113, 2056-2075.

[5] J. E. Moses, A. D. Moorhouse, Chem. Soc. Rev. 2007, 36, 1249-1262.

[6] M. Wei, D. Liang, X. Cao, W. Luo, G. Ma, Z. Liu, L. Li, Angew. Chem. Int Ed. 2020, DOI: 10.1002/anie.202013976.

[7] J.-N. Luy, R. Tonner, ACS Omega 2020, 5, 31432-31439.

[8] S. Mahapatra, C. P. Woroch, T. W. Butler, S. N. Carneiro, S. C. Kwan, S. R. Khasnavis, J. Gu, J. K. Dutra, B. C. Vetelino, J. Bellenger, C. W. am Ende, N. D. Ball, Org. Lett. 2020, 22, 4389-4394.

[9] B. Gao, S. Li, P. Wu, J. E. Moses, K. B. Sharpless, Angew. Chem. Int. Ed. 2018, 57, 1939-1943; Angew. Chem. 2018, 130, 1957-1961.

[10] C. J. Smedley, Q. Zheng, B. Gao, S. Li, A. Molino, H. M. Duivenvoorden, B. S. Parker, D. J. D. Wilson, K. B. Sharpless, J. E. Moses, Angew. Chem. Int. Ed. 2019, 58, 4552-4556; Angew. Chem. 2019, 131, 4600-4604.

[11] T. Kalliokoski, ACS Comb. Sci. 2015, 17, 600-607.

[12] G. Meng, T. Guo, T. Ma, J. Zhang, Y. Shen, K. B. Sharpless, J. Dong, Nature 2019, 574, 86-91.

[13] S. Li, P. Wu, J. E. Moses, K. B. Sharpless, Angew. Chem. Int. Ed. 2017 56, 2903-2908; Angew. Chem. 2017, 129, 2949-2954. 
[14] M.-C. Giel, C. J. Smedley, E. R. R. Mackie, T. Guo, J. Dong, T. P. Soares da Costa, J. E. Moses, Angew. Chem. Int. Ed. 2020, 59, 1181-1186; Angew. Chem. 2020, 132, 1197-1202.

[15] C. J. Smedley, M.-C. Giel, A. Molino, A. S. Barrow, D. J. D. Wilson, J. E. Moses, Chem. Commun. 2018, 54, 6020-6023.

[16] J. Leng, H.-L. Qin, Chem. Commun. 2018, 54, 4477-4480.

[17] J. Thomas, V. V Fokin, Org. Lett. 2018, 20, 3749-3752.

[18] C. J. Smedley, G. Li, A. S. Barrow, T. L. Gialelis, M.-C. Giel, A. Ottonello, Y. Cheng, S. Kitamura, D. W. Wolan, K. B. Sharpless, J. E. Moses, Angew. Chem. Int. Ed. 2020, 59, 12460-12469; Angew.Chem. 2020, 132, 12560-12569.

[19] V. Gembus, F. Marsais, V. Levacher, Synlett 2008, 1463-1466.

[20] A. S. Barrow, J. E. Moses, Synlett 2016, 27, 1840-1843.

[21] J. Dong, K. B. Sharpless, L. Kwisnek, J. S. Oakdale, V. V. Fokin, Angew. Chem. Int. Ed. 2014, 53, 9466-9470; Angew.Chem. 2014, 126, 9620 9624.

[22] J. Yatvin, K. Brooks, J. Locklin, Angew. Chem. Int. Ed. 2015, 54, 13370 13373; Angew. Chem. 2015, 127, 13568-13571.

[23] B. Gao, L. Zhang, Q. Zheng, F. Zhou, L. M. Klivansky, J. Lu, Y. Liu, J. Dong, P. Wu, K. B. Sharpless, Nat. Chem. 2017, 9, 1083-1088.

[24] A. M. Hyde, R. Cal, Org. Process Res. Dev. 2019, 23, 1860-1871.

[25] D.-D. Liang, D. E. Streefkerk, D. Jordaan, J. Wagemakers, J. Baggerman, H. Zuilhof, Angew. Chem. Int. Ed. 2020, 59, 7494-7500; Angew. Chem. 2020, 132, 7564-7570.

[26] C. Li, Y. Zheng, K. P. Rakesh, H.-L. Qin, Chem. Commun. 2020, 56, 8075-8078.

[27] D. H. R. Barton, J. D. Elliott, S. D. Géro, J. Chem. Soc. Perkin Trans. 1 1982, 2085-2090.

[28] D. H. R. Barton, B. Charpiot, W. B. Motherwell, Tetrahedron Lett. 1982 , 23, 3365-3368.

[29] Trimethylsilanol (TMSOH, 20.0 eq.) has been employed as a post-SuFEx quench to sequester the fluoride ion product as the volatile fluorotrimethylsilane (TMSF, b.p. is $16^{\circ} \mathrm{C}$ ), see: Z. Liu, J. Li, S. Li, G. Li, K. B. Sharpless, P. Wu, J. Am. Chem. Soc. 2018, 140, 2919-2925.

[30] The high reactivity of silyl ethers in acetonitrile is ascribed to that solvent's good coordinating properties with silicon; see [19] and references therein.

[31] C. Liu, C. Yang, S. Hwang, S. L. Ferraro, J. P. Flynn, J. Niu, Angew. Chem. Int. Ed. 2020, 59, 18435-18441; Angew. Chem. 2020, 132, 18593-18599.

[32] Dr. A. S. Barrow was the first to perform a model SuFEx reaction using BTMG as a catalyst with $\mathrm{TMSOH}$ additive in the Moses laboratory (November 19 ${ }^{\text {th }}, 2019$, unpublished results).

[33] A. G. M. Barrett, S. M. Cramp, R. S. Roberts, F. J. Zecri, Org. Lett. 1999, 1, 579-582.

[34] A. G. M. Barrett, S. M. Cramp, A. J. Hennessy, P. A. Procopiou, R. S. Roberts, Org. Lett. 2001, 3, 271-273.

[35] P. Wipf, S. M. Lynch, Org. Lett. 2003, 5, 1155-1158.

[36] J. E. Perea-Buceta, I. Fernández, S. Heikkinen, K. Axenov, A. W. T. King, T. Niemi, M. Nieger, M. Leskelä, T. Repo, Angew. Chem. Int. Ed. 2015, 54, 14321-14325; Angew.Chem. 2015, 127,14529-14533.

[37] H. Kotsuki in Superbases for Organic Synthesis. Guanidines, Amidines, Phosphazenes and Related Organocatalysts (Ed. T. Ishikawa), John Wiley \& Sons, Ltd., 2009, pp. 187.

[38] The hydrolysis of HMDS gives the volatile trimethylsilanol (TMSOH) and ammonia $\left(\mathrm{NH}_{3}\right)$; for example, see R. Pellegata, A. Italia, M. Villa Synthesis 1985, 517-519.

[39] M. Epifanov, P. J. Foth, F. Gu, C. Barrillon, S. S. Kanani, C. S. Higman, J. E. Hein, G. M. Sammis, J. Am. Chem. Soc. 2018, 140, 16464-16468.

[40] A. A. Joseph, V. P. Verma, X.-Y. Liu, C.-H. Wu, V. M. Dhurandhare, C.C. Wang, European J. Org. Chem. 2012, 2012, 744-753.

[41] The ${ }^{1} \mathrm{H}$ NMR spectra in Figure 2 shows the disappearance of water upon addition of HMDS; see [38]

[42] The DBU-mediated de-silylation of aryl silyl ethers has been reported: C. -E. Yeom, H. W. Kim, S. Y. Lee, B. M. Kim, Synlett 2007, 146-150.

[43] B. Soto-Cairoli, I. Kock, J. J. de Pomar, G. Yang, J. M. Guzmán, J. R. González, A. Antomattei, J. A. Soderquist, Heterocycles 2010, 80, 409426. 